\title{
Surface electromyographic analysis of differential effects in kettlebell carries for the serratus anterior muscles
}

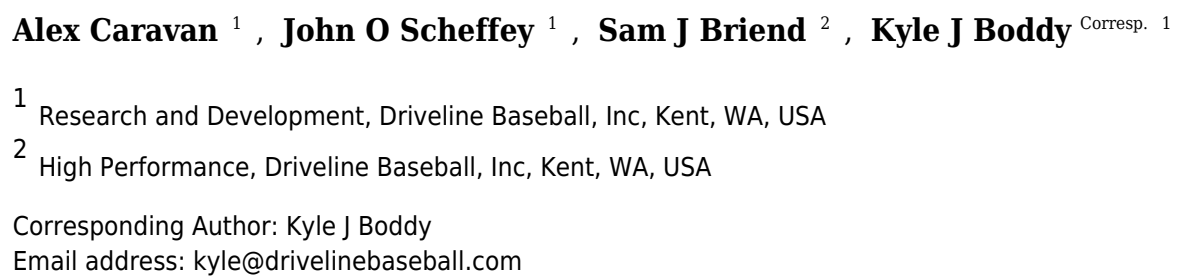

Abstract The purpose of this study was to examine differences in the Electromyography (EMG) amplitude of the serratus anterior between forty-five degree kettlebell carries and ninety degree kettlebell carries. Thirty-three men aged roughly between 19-23 and who were either college or professional baseball pitchers were chosen and randomly assigned to either perform the forty-five degree kettlebell carry followed by the ninety degree kettlebell carry $(n=17)$ or the ninety degree kettlebell carry followed by the forty five degree kettlebell carry $(n=16)$. Each pitcher was instructed in the proper usage of the exercise and assigned a short break between the two carries. Changes in EMG amplitude were examined after proper band-pass filtering, normalization, and moving averagesmoothing of the raw EMG signal. Differences of the EMG amplitude mean frequencies were examined between each subject's individual carries and the clumped groups of all forty-five and ninety degree carries. Among each individual comparison, eight pitchers had "large" Effect Size differences between the EMG amplitudes of their two carries, with seven of them signaling the forty-five degree carry as the larger value. In addition, when examining the grouped mean differences of the EMG amplitudes, we found the forty-five degree carries to be significantly higher ( $p$-value of 0.018 ). 


\section{Surface electromyographic analysis of differential effects in 2 kettlebell carries for the serratus anterior muscles}

3 Alex Caravan $^{1}$, John O. Scheffey ${ }^{2}$, Sam J. Briend ${ }^{3}$, Kyle J. Boddy ${ }^{4}$

$4 \quad 1,2,4$ Driveline Baseball, Research \& Development, Kent, WA USA

$5 \quad{ }^{3}$ Driveline Baseball, High Performance, Kent, WA USA

6 Corresponding Authors:

7 Sam J. Briend, Kyle J. Boddy

819612 70th Avenue South, Unit 2-4

9 Kent, WA 98032

10 Email addresses: sam@drivelinebaseball.com, kyle@drivelinebaseball.com 
12

\section{Abstract}

The purpose of this study was to examine differences in the Electromyography (EMG) amplitude of the serratus anterior between forty-five degree kettlebell carries and ninety degree kettlebell carries. Thirty-three men aged roughly between 19-23 and who were either college or professional baseball pitchers were chosen and randomly assigned to either perform the fortyfive degree kettlebell carry followed by the ninety degree kettlebell carry $(n=17)$ or the ninety degree kettlebell carry followed by the forty five degree kettlebell carry $(n=16)$. Each pitcher was instructed in the proper usage of the exercise and assigned a short break between the two carries. Changes in EMG amplitude were examined after proper band-pass filtering, normalization, and moving average-smoothing of the raw EMG signal. Differences of the EMG amplitude mean frequencies were examined between each subject's individual carries and the clumped groups of all forty-five and ninety degree carries. Among each individual comparison, eight pitchers had "large" Effect Size differences between the EMG amplitudes of their two carries, with seven of them signaling the forty-five degree carry as the larger value. In addition, when examining the grouped mean differences of the EMG amplitudes, we found the forty-five degree carries to be significantly higher ( $p$-value of 0.018 ).

\section{Introduction}

For overhead athletes poor scapula function can result in a myriad of issues, the least of which are adverse force generation and a propensity for shoulder injury (Myers et al., 2005). To counter these detrimental effects for overhead athletes' bottoms-up kettlebell carries, or kettlebell carries, are commonly used as a recovery, rehabilitation and strength training exercise. Activation of the serratus anterior is the intended focus of these exercises as it is a prime mover for both scapular upward rotation and scapula stabilization, especially during overhead movements (Phadke, Camargo \& Ludewig, 2009). In turn, these movements help limit subacromial impingement and stabilize the scapula (Ludeqig \& Cook, 2000).

However, to our knowledge, no studies exist that examine muscle activation of overhead stabilizers like the serratus anterior during kettlebell carries. Several variations of the kettlebell carry are commonly used, with little data comparing the effectiveness of different variations.

This study involved measurements of EMG amplitude to examine differences across various positions and exercises, following similar precedent from past studies (Decker et al. 1999). EMG is commonly used to measure electric potential differences caused by muscle activation. However, because it can be affected by various forms of noise, including equipment calibration, background electromagnetic radiation, movement of electrodes, randomness of muscle firing patterns, and electrocardiography (ECG) signal, certain to-be-discussed analytical methods were 
50

51

52

53

54

55

56

57

58

59

60

61

62

63

64

65

66

67

68

69

70

71

72

73

74

75

76

77

78

79

80

81

82

83

84

85

86

87

88

89

undertaken to maximize the bifurcation of the signal and the noise including the filtering, normalization, and smoothing of the raw EMG signal.

The purpose of this study was to use EMG sensors to examine serratus anterior activation in baseball pitchers performing bottoms up kettlebell carries at 90 degrees and approximately 45 degrees of horizontal abduction of the humerus, and to determine if there is a statistically significant difference in the EMG amplitude of the serratus anterior between the two variations. Zero degrees was set as the arm being extended in front of the person, and 90 degrees was set as the arm being extended out to the side (Figure 1, Figure 2). Kettlebell carry variations that cause a higher serratus anterior amplitude may imply higher muscle activation and may be more beneficial for the recovery and rehabilitation of overhead throwing athletes.

[Figure 1]

[Figure 2]

\section{Methods}

\section{Subjects}

The study was approved by Hummingbird IRB, who granted ethical approval to carry out the study (Hummingbird IRB\# 2017-58) at the author's facilities. Subjects were provided a verbal explanation of the study and then given an Informed Consent form to read and sign before participating. Forty healthy college and professional pitchers training at the Driveline Baseball facility in Kent, Washington were selected as participants in the study. Due to failure to follow instructions and mismatched data this number was reduced, with thirty-three total subjects having their data included in the final analysis (age $=22.2 \pm 3.5$ years; height $=73.4 \pm 2.1$ inches; body weight $=200.8 \pm 17.6 \mathrm{lbs}$ ). Inclusion criteria required subjects to be between 18 and 40 years of age and not possess a significant medical history of shoulder or rotator cuff injuries. Subjects were required to be able to lift at least $50 \mathrm{lbs}$ from the ground without discomfort or pain in the upper extremities, specifically the forearm, elbow, shoulder, and neck areas. If pain or discomfort was experienced at any point during trials, the study was stopped and the participant's data were not included.

\section{Procedures}

Subjects were recruited to participate in the study after throwing, as part of their post-training recovery exercises. The exercise is intended to serve as a post-throwing recovery exercise in order to reduce risk of arm pain and instability. Once ready to participate, subjects were selected 
90

91

92

93

94

95

96

97

98

99

100

101

102

103

104

105

106

107

108

109

110

111

112

113

114

115

116

117

118

119

120

121

122

123

124

125

126

127

128

129

from the general population and placed in the two distinct groups of twenty subjects through a block randomization process. The serratus anterior of the dominant arm was cleaned and a Somaxis Cricket EMG sensor (Somaxis Inc., San Francisco, CA, USA) was applied vertically across a standardized site on the serratus anterior. (Figure 3) The EMG sensor was held in place using kinesiology tape (RockTape, Inc).

\section{[Figure 3]}

Prior to performing kettlebell carries, maximum voluntary isometric contraction (MVIC) data for each subject was collected, to normalize future EMG readings for the kettlebell carries. Subjects were instructed to raise their dominant arm to 125 degrees of shoulder abduction in the scapular plane (not to be confused with the previous references to the horizontal humeral plane) and a goniometer was used to measure the exact range of motion. Subjects were then instructed, with their arm raised in the same measured position, to resist a downward force of 5 pounds applied to the humerus with a hand-held dynamometer (Lafayette Instrument $\mathrm{Co}$ ) by an experimenter, to achieve MVIC. (Figures 4, 5) Manual resistance at a shoulder abduction angle of about 120 degrees has been shown to achieve higher serratus anterior activation than other MVIC positions (Jung and Moon, 2015).

\section{[Figure 4]}

\section{[Figure 5]}

Subjects were then coached in the proper technique for kettlebell carries with a standardized set of instructions. The first group performed a bottoms-up kettlebell carry with the humerus at 90 degrees of horizontal abduction and the forearm vertical to the floor. (Figure 1) After completing the first carry, subjects took a short break of around 30 seconds and then were told to complete a second kettlebell carry trial with the humerus at 45 degrees of horizontal abduction and the forearm vertical to the floor. (Figure 2) The second group performed the same two carries, in reverse order.

Weight and distance for the kettlebell carries was standardized at $25 \mathrm{lbs}$ for the kettlebell and 30 yards for the distance, with a turnaround at 15 yards. The 15 yard mark was clearly defined with tape, and subjects were instructed to walk at a comfortable pace. Subjects were instructed to maintain upright posture with shoulders parallel to the floor during the carries.

The 25 lbs was chosen as the standardized weight as the kettlebell carry is often performed as a recovery exercise in-gym for many of the athletes, and the individual athlete exercises discretion in choosing weights ranging from $15 \mathrm{lbs}$ to $35 \mathrm{lbs}$; likewise, the 30 yards is the default standardized distance used during in-gym exercise. 
130

131

132

133

134

135

136

137

138

139

140

141

142

143

144

145

146

147

148

149

150

151

152

153

154

155

156

157

158

159

160

161

162

163

164

165

166

167

168

Raw EMG signals were collected at $1,000 \mathrm{~Hz}$ by a Somaxis Cricket EMG sensor (Somaxis Inc., San Francisco, CA, USA). Data were sent in real time to an iPhone via Bluetooth and recorded by the Chirp for Cricket application (Somaxis Inc., San Francisco, CA, USA).

\section{Statistical Analyses}

The EMG sensor generated two columns of data for each pitcher, one containing the raw data and the second returning data from a specialized built-in cricket filter. For the sake of scientific test-re-test reliability and an open source protocol, the raw data was used. After disallowing a few aforementioned subjects, the remaining thirty-three subjects compromised seventeen subjects who performed the 45 degree carry followed by the 90 degree carry (the first group) and sixteen subjects who performed the 90 degree carry followed by the 45 degree carry (the second group).

As referenced above, surface EMG cannot and should not be used to implicate muscle activation; instead, EMG measures changes in the force-generation magnitude of the individual muscle fibers' membranes rather than the quantity of active muscle fibers, which is what generates muscle activation (Vigotsky et al., 2017). As such, EMG amplitude is more accurately a measure of muscle excitation. Even with these careful denotations, a number of filtering, normalizing, and smoothing measures were taken to clean the raw EMG data.

First, a band pass filter was used to remove components below and above selected frequency cutoffs of $10 \mathrm{HZ}$ (the low end cutoff, traditionally used to remove electrical noise associated with wire sway, biological artifacts etc.) and $450 \mathrm{~Hz}$ (the high end cutoff, used to eliminate tissue noise at the electrode site) (De Luca et al. 2010).

These cutoff frequencies were then applied with a Butterworth filter to transform each of the pitchers raw data into a filtered output, for better detection of the onset and offset of the kettlebell carries. A detection algorithm based on a double threshold scheme was applied to detect the start and end of the kettlebell carries in each pitchers' continuous EMG data (Bonato et al. 1998). After these carries were identified, they were normalized with the peak MVIC value for each respective subject and smoothed with a moving-average based envelope of the normalized signal for further elimination of any phase shifts of the signal. Normalization of the signal, even post filtering, is required to enable a valid comparison between different individuals and different tasks (Halaki and Ginn, 2012). Per past EMG literature, the optimal window size of 200 was used for the said-MA smoothing (Dietrich et al., 2017).

Statistical analysis was done on both a) intra-subject differences for the each of the 33 pairs of carries and b) the group differences between all the 45 degree carries and all the 90 degree carries (Phinyomark et al., 2008). Analysis revolved around using the mean frequencies and median frequencies of the EMG filtered signal because frequency-domain features have been shown to be the most ideal variables in identifying muscle fatigue and force (Phinyomark et al., 2012). 
169

170

171

172

173

174

175

176

177

178

179

180

181

182

183

184

185

186

187

188

189

190

191

192

193

194

195

196

197

198

199

200

201

202

203

204

205

For the intra-subject comparisons, three different avenues of statistical comparisons were pursued: a) the Effect Size Test (specifically, the Cohen's D estimate) as a method of statistical power analysis that looks at the standardized difference between means; b) the Granger Test, which pinpoints predictive causality of the first carry towards the second one; and c) the normalized distance from a dynamic time warping method that relies on minimizing the Euclidean distance off an optimally aligned time series. (Figure 6)

[Figure 6]

\section{Results}

Out of all the 33 individuals, 22 had the larger mean frequency in the 45 degree kettlebell carry. Splitting the groups by the order of carries, the first group ( 90 degree carry performed first) found 10/16 subjects with a higher value in the 45 degree carry, while the second group found $12 / 17$ subjects with the higher value in said 45 degree carry. Judging by the Cohen D statistics (where the widely accepted scales of significance are 0.2 to $0.5 d$ for a "small" effect, 0.5 to $0.8 d$ for a "medium" effect and anything above $0.8 d$ is deemed as a "large" effect), 8 subjects had a large effect ( 7 of them with the higher mean in the 45 degree carry), 8 subjects had a medium effect ( 4 of them in the 45 degree carry), and 10 subjects had a small effect ( 6 in the 45 degree carry) (Sawilowksy, 2009).

Looking at the Granger Test of forecasting probability (with a chosen alpha level of 0.05), the majority of the subjects had a significantly predictive first carry, with 10 of these 17 significant Granger estimates registering among the group that performed the 45 degree carry first.

Lastly, we looked at the normalized distance between the individuals where the Euclidean cost was minimized via the dynamic time warping alignment, as a method of verifying the Effect Size denoted by Cohen's D. (Table 1) We found the "large" effects averaged a dissimilarity measure of 2.53, the medium effects averaged 1.60, and the small effects averaging around 1.00 (the differences that didn't register interestingly had around a 1.23 average).

\section{[Table 1]}

Looking at the holistic groups of 45 degree and 90 degree carries, a paired $\mathrm{T}$ test was used to judge the significance of the difference between the two groups of mean frequencies. (Table 2) The paired T test was chosen because the sample size was large enough to guarantee a normal distribution; in addition, the plot density and quantile-quantile plots all showed normality for both groups of means, while both groups comfortably failed to reject the Shapiro Test of normality (registering p-values of 0.879 and 0.791 , where a sub 0.05 score would have been needed to reject the null hypothesis of normality). Furthermore, even the order-dependent 
206

207

208

209

210

211

212

213

214

215

216

217

218

219

220

221

222

223

224

225

226

227

228

229

230

231

232

233

234

235

236

237

238

239

240

241

242

243

subgroups of the means (the 45 degree and 90 degree carries of both the first and second group) fulfilled all the aforementioned normality checks.

[Table 2]

The Paired T Test returned a significant value of 0.0180 , with the mean frequencies of the groups being 9.799 and 9.354 for the 45 degree and 90 degree carries, respectively. The difference stems in larger part from the subjects who performed the 45 degree carry first, as a mean difference analysis of that subgroup returned a significant $p$ level by itself $(0.0325$ versus a $p$ level of 0.246 from the subgroup who performed the 45 degree carries second).

\section{Discussion}

The purpose of this investigation was to examine differences in the muscle engagement of the serratus anterior between 45 degree and 90 degrees kettlebell carries. These differences were examined across both intra-subject carries and the overall groups of carry mean frequencies. While the intra-subject differences ranged from inconsequential differences to "large effect" differences, the grouped mean difference analysis returned a steady significant result of a 0.018 p-value.

As mentioned above, a number of considerations have to be taken in account in the emerging world of EMG analysis and statistical studies. Specifically, for the scope of this study the main concern resides in drawing inferences about how closely EMG amplitude indicates agonist muscle activation, as the raw signal of the EMG amplitude is often muddied by noise from the subject's unique muscle fiber recruitment and motor unit firing frequency (Kuriki et al. 2012), as well as the exact location of the EMG sensor and the quantity of the respective subcutaneous tissue on which it is placed (De Luca, 1997). Hence, the investigation adopted proper filtering (Stegeman \& Hermens, 2014), normalization (Alkner et al., 2000), and frequency extraction (Phinyomark et al., 2012).

There have been several studies concerning the EMG activity of the serratus anterior, although none have dealt with the exact nature of this study's investigation. A study examining variations in the EMG activity of different muscles throughout the push-up plus exercise found that SA activity was significantly greater during the "plus" phase of the exercise and was very malleable to different hand positioning and support surfaces (Gioftsos et al., 2016).

In addition, and perhaps more relevantly to this study's design, a 2003 study measured the EMG activity of separate trapezius muscles and the serratus anterior muscles across ten different scapula-involved exercises (Ekstrom et al., 2003). It found that the highest EMG activations were achieved by, in order, the (a) Diagonal exercise with shoulder flexion, horizontal flexion, and external rotation and (b) the Shoulder abduction in the plane of the scapula above $120^{\circ}$, the two exercises most similar in movement to a 45 degree and 90 degree kettlebell carry, 
244

245

246

247

248

249

250

251

252

253

254

255

256

257

258

259

260

261

262

263

264

265

266

267

268

269

270

271

272

273

274

275

276

277

278

279

280

281

respectively. However, our findings were in contrary to the study, as we found the differences of the EMG amplitudes significant, while the aforementioned study found a nonsignificant difference. It's important to note though, that the 2003 study involved submaximal voluntary dynamic contraction (SVDC) of the exercises versus the submaximal voluntary isometric contraction (SVIC) movements that were observed in this study.

Future studies could explore a number of things. The study could be reproduced before a workout rather than after, in case a pre-fatigue MVIC (and subsequent SVIC) generates different results. There could be further variations in the degree of the angle abduction of the kettlebell carry: between 0 and 45 degrees and between 45 degrees and 90 degrees. There could also be more variability in the weights used, to see if the differences change in magnitude with the weight used, or even reverses. Further, there could be investigations into prior postural deficiencies or upper body mobility and flexibility capabilities, to try to control for differences in biomechanical advantages impacting just how much an individual relies on or stresses their serratus. A longitudinal study would be especially interesting in exploring how the serratus activation changes in behavior with the subject's own body and physical attributes.

\section{Conclusion}

Our findings indicated that there is a significant increase in EMG amplitude for the serratus anterior in the forty-five degree kettlebell carry versus the complementary ninety degree one for a population of adult male pitchers. For various reasons, it is hard to draw an exact match between muscle activation and EMG amplitude, but it appears likely that muscle activation of the serratus anterior is higher in the case of the former exercise. These findings can be used to increase the chances of athletes retaining arm health by offering forty-five degree kettlebell carries as part of a thorough post-workout recovery exercise routine.

\section{References}

Myers JB., Laudner KG., Pasquale MR., Bradley JP., Lephart SM. 2005. Scapular Position and Orientation in Throwing Athletes. The American Journal of Sports Medicine 33:263-271. DOI: $10.1177 / 0363546504268138$.

Phadke V., Camargo P., Ludewig P. 2009. Scapular and rotator cuff muscle activity during arm elevation: A review of normal function and alterations with shoulder impingement. Revista brasileira de fisioterapia (Sao Carlos (Sao Paulo, Brazil)) 13:1-9. DOI: 10.1590/S141335552009005000012.

Ludewig PM., Cook TM. 2000. Alterations in Shoulder Kinematics and Associated Muscle Activity in People With Symptoms of Shoulder Impingement. Physical Therapy 80:276-291. DOI: $10.1093 / \mathrm{ptj} / 80.3 .276$.

Decker MJ., Hintermeister RA., Faber KJ., Hawkins RJ. 1999. Serratus Anterior Muscle Activity During Selected Rehabilitation Exercises. The American Journal of Sports Medicine 27:784791. DOI: 10.1177/03635465990270061601. 
282 Kuriki, H. U., Mello, E. M., De Azevedo, F. M., Takahashi, L. S. O., Alves, N., \& de Faria

283 Negrão Filho, R. (2012). The relationship between electromyography and muscle force.INTECH

284 Open Access Publisher

285 De Luca, C. J. (1997). The use of surface electromyography in biomechanics. Journal of applied

286 biomechanics, 13, 135-163

287 Jung D., Moon D. 2015. The effects of shoulder joint abduction angles on the muscle activity of

288 the serratus anterior muscle and the upper trapezius muscle while vibrations are applied. Journal

289 of Physical Therapy Science 27:117-119. DOI: 10.1589/jpts.27.117.

290 Vigotsky AD., Halperin I., Lehman GJ., Trajano GS., Vieira TM. 2018. Interpreting Signal

291 Amplitudes in Surface Electromyography Studies in Sport and Rehabilitation Sciences. Frontiers

292 in Physiology 8:985. DOI: 10.3389/fphys.2017.00985.

293 De Luca CJ., Donald Gilmore L., Kuznetsov M., Roy SH. 2010. Filtering the surface EMG

294 signal: Movement artifact and baseline noise contamination. Journal of Biomechanics 43:1573-

295 1579. DOI: 10.1016/j.jbiomech.2010.01.027.

296 P. Bonato, T. D'Alessio and M. Knaflitz, "A statistical method for the measurement of muscle

297 activation intervals from surface myoelectric signal during gait," in IEEE Transactions on

298 Biomedical Engineering, vol. 45, no. 3, pp. 287-299, March 1998.

299 Mark Halaki and Karen Ginn (2012). Normalization of EMG Signals: To Normalize or Not to

300 Normalize and What to Normalize to?, Computational Intelligence in Electromyography

301 Analysis - A Perspective on Current Applications and Future Challenges, Dr. Ganesh R. Naik

302 (Ed.), InTech, DOI: 10.5772/49957.

303 Dieterich, A. V., Botter, A., Vieira, T. M., Peolsson, A., Petzke, F., Davey, P., Falla, D. (2017).

304 Spatial variation and inconsistency between estimates of onset of muscle activation from EMG

305 and ultrasound. Sci. Rep. 7:42011. doi: 10.1038/srep42011

306 Phinyomark A., Thongpanja S., Hu H., Phukpattaranont P., Limsakul C. 2012. The Usefulness of

307 Mean and Median Frequencies in Electromyography Analysis. In: Naik GR ed. Computational

308 Intelligence in Electromyography Analysis - A Perspective on Current Applications and Future

309 Challenges

310 Angkoon Phinyomark, Sirinee Thongpanja, Huosheng Hu, Pornchai Phukpattaranont and

311 Chusak Limsakul (2012). The Usefulness of Mean and Median Frequencies in

312 Electromyography Analysis, Computational Intelligence in Electromyography Analysis - A

313 Perspective on Current Applications and Future Challenges, Dr. Ganesh R. Naik (Ed.), InTech,

314 DOI: $10.5772 / 50639$.

315 Sawilowsky, Shlomo S. (2009) "New Effect Size Rules of Thumb," Journal of Modern Applied

316 Statistical Methods: Vol. 8 : Iss. 2 , Article 26. DOI: 
317 Standards for surface electromyography: the European project "Surface EMG for non-invasive 318 assessment of muscles (SENIAM)", Stegeman \& Hermens. Online: http://www.med.uni319 jena.de/motorik/pdf/stegeman.pdf, retrieved 2014-02-19.

320 Alkner, B. A., Tesch, P. A., and Berg, H. E. (2000). Quadriceps EMG/force relationship in knee 321 extension and leg press. Med. Sci. Sports Exerc. 32, 459-463. doi: 10.1097/00005768-

$322 \quad 200002000-00030$

323 Gioftsos G., Arvanitidis M., Tsimouris D., Kanellopoulos A., Paras G., Trigkas P., Sakellari V. 324 EMG activity of the serratus anterior and trapezius muscles during the different phases of the 325 push-up plus exercise on different support surfaces and different hand positions. Journal of 326 Physical Therapy Science. 2016;28(7):2114-2118. doi:10.1589/jpts.28.2114.

327 Surface Electromyographic Analysis of Exercises for the Trapezius and Serratus Anterior 328 Muscles 2003. Journal of Orthopaedic \& Sports Physical Therapy 33:247-258. DOI: $329 \quad 10.2519 /$ jospt.2003.33.5.247. 


\section{Figure 1}

\section{Kettlebell carry at 90 degrees}

An example of a subject carrying the kettlebell at approximately 90 degrees of horizontal shoulder abduction.

Photo credit: John O Scheffey 


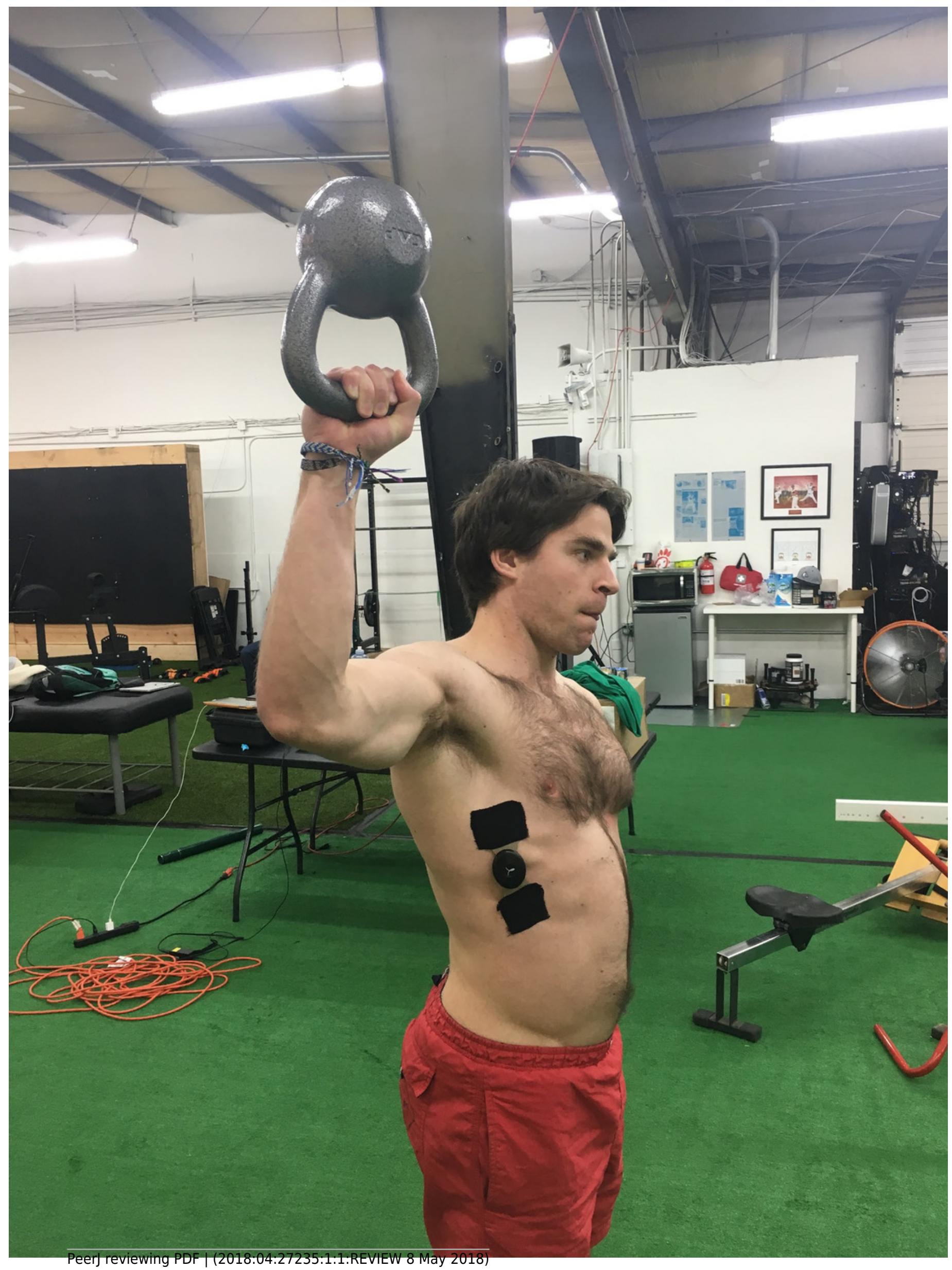




\section{Figure 2}

\section{KB carry at 45 degrees}

An example of a subject carrying the kettlebell at approximately 45 degrees of horizontal shoulder abduction.

Photo credit: John O Scheffey 


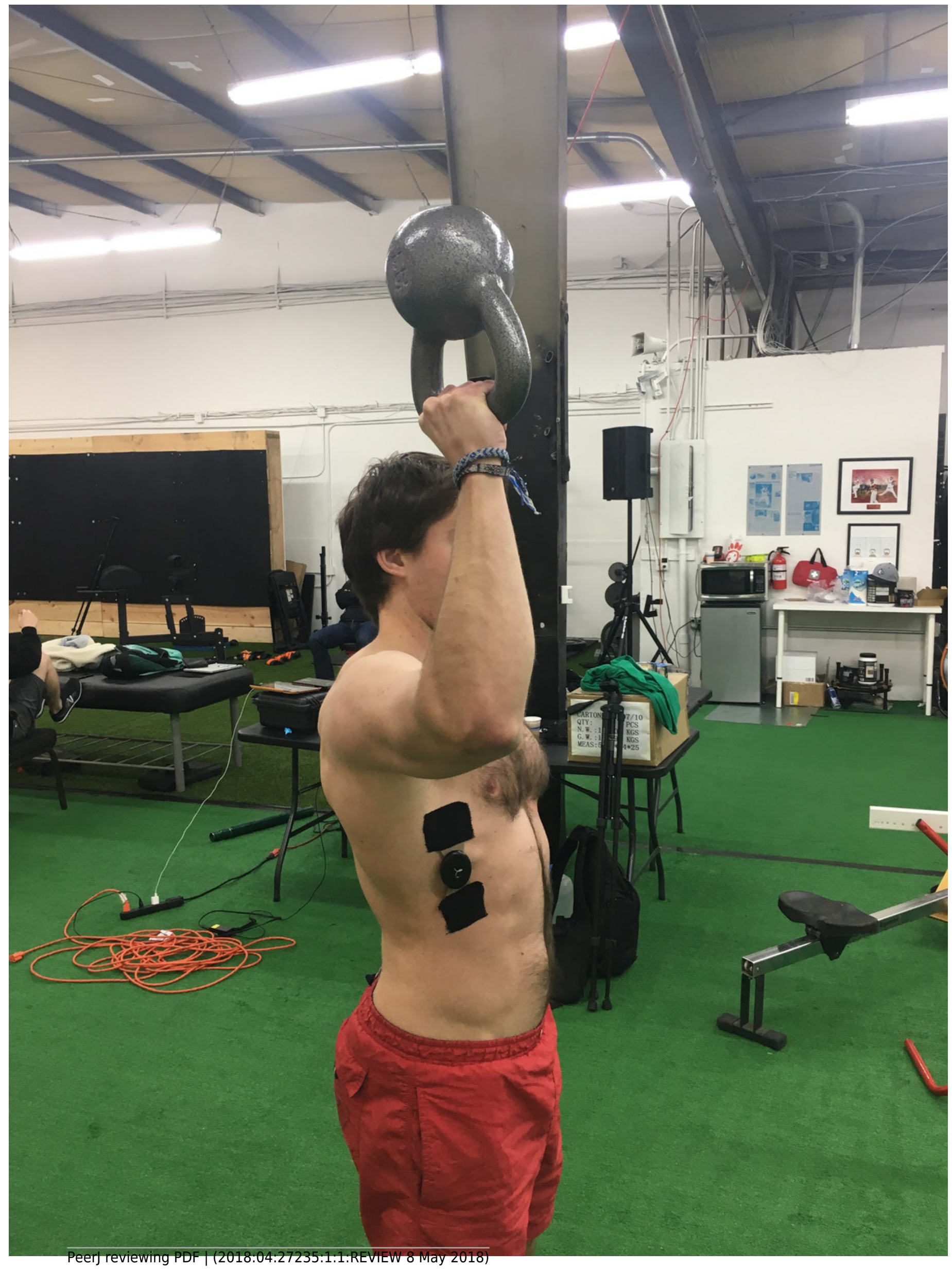




\section{Figure 3}

\section{EMG application example}

Application of the Somaxis EMG sensor to the subject.

Photo credit: John O Scheffey 


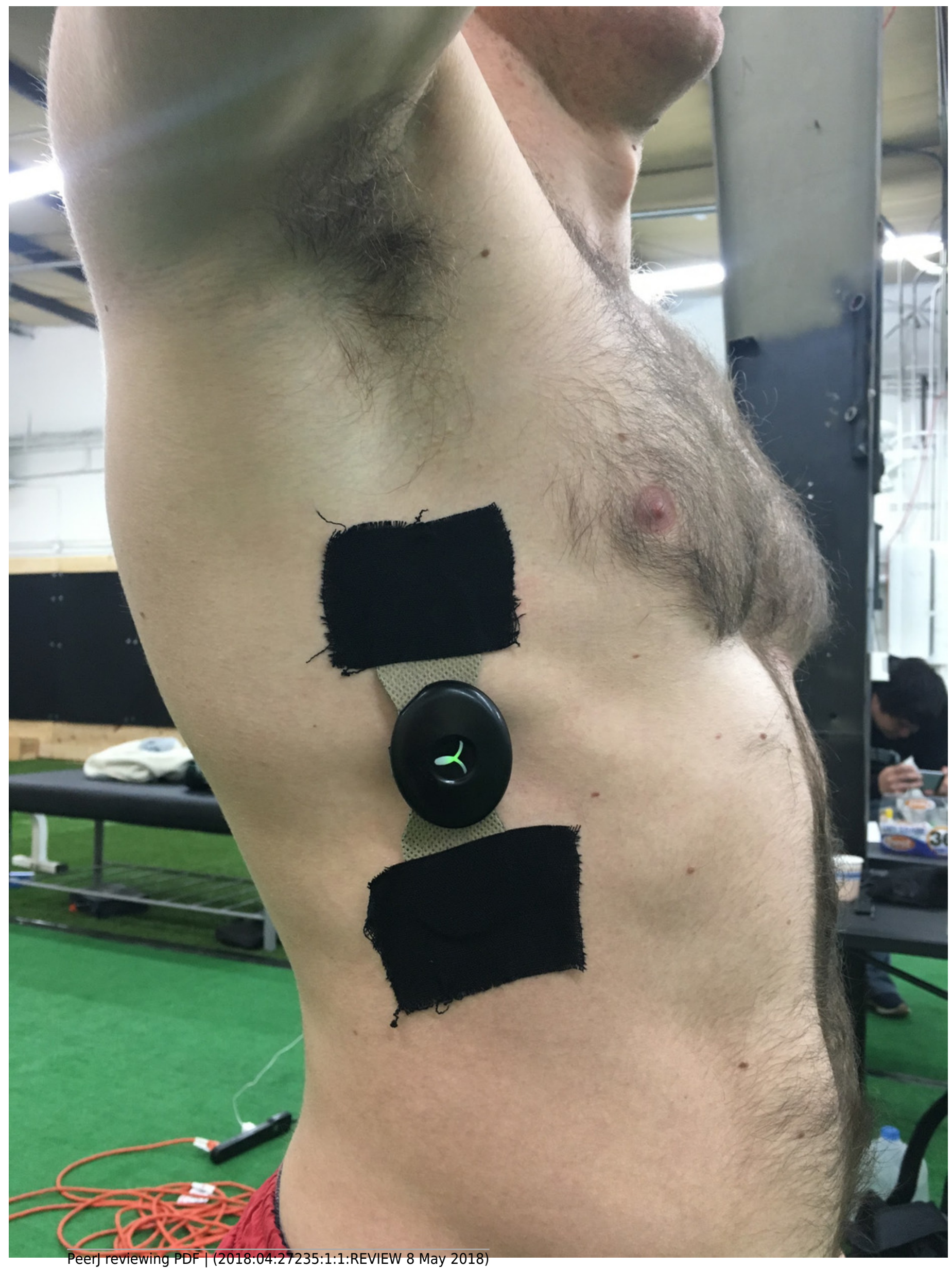




\section{Figure 4}

\section{MVIC test 1}

The first MVIC test done on subjects.

Photo credit: John O Scheffey

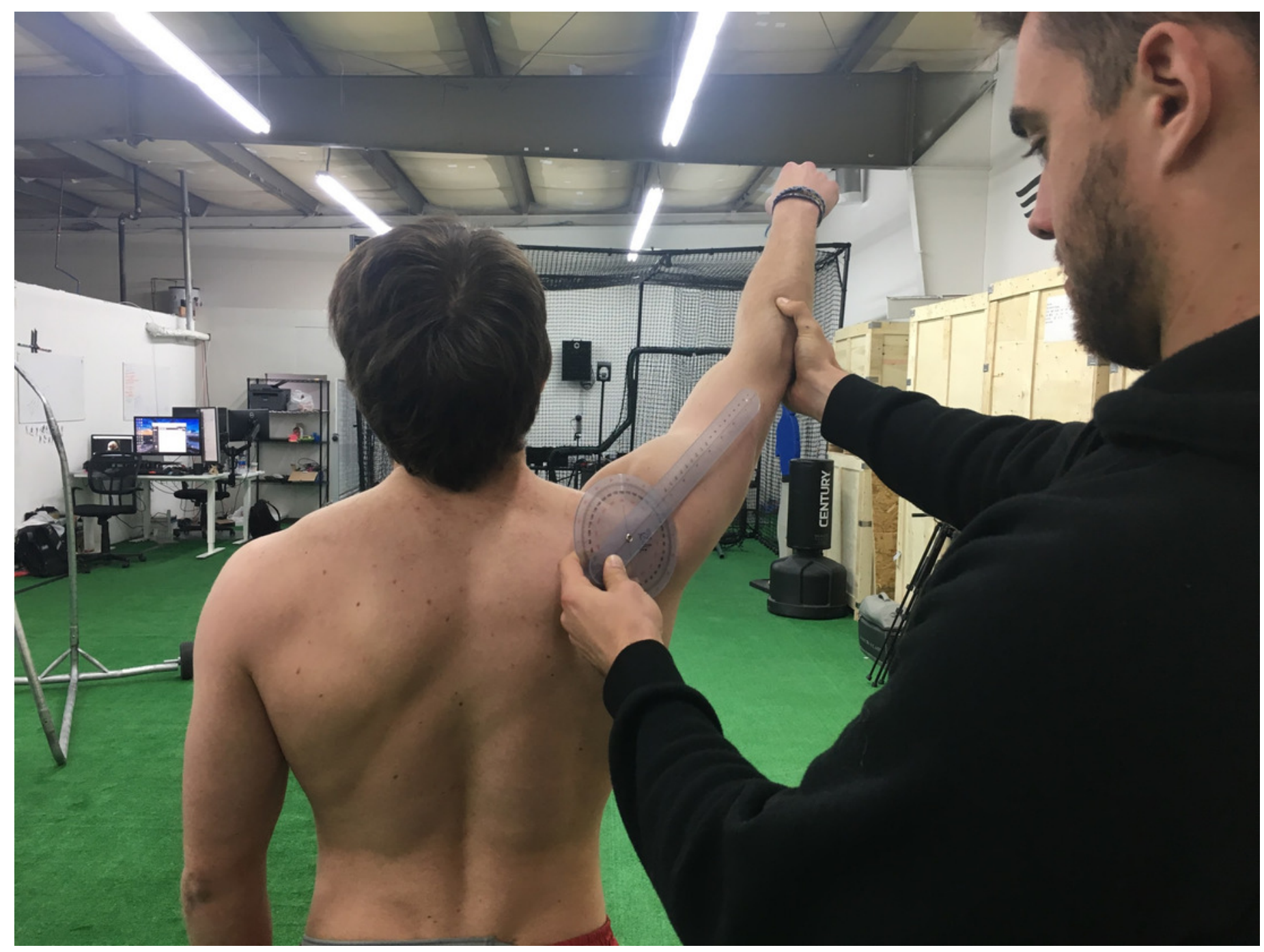




\section{Figure 5}

\section{MVIC test 2}

The second image in the MVIC test series.

Photo credit: John O Scheffey

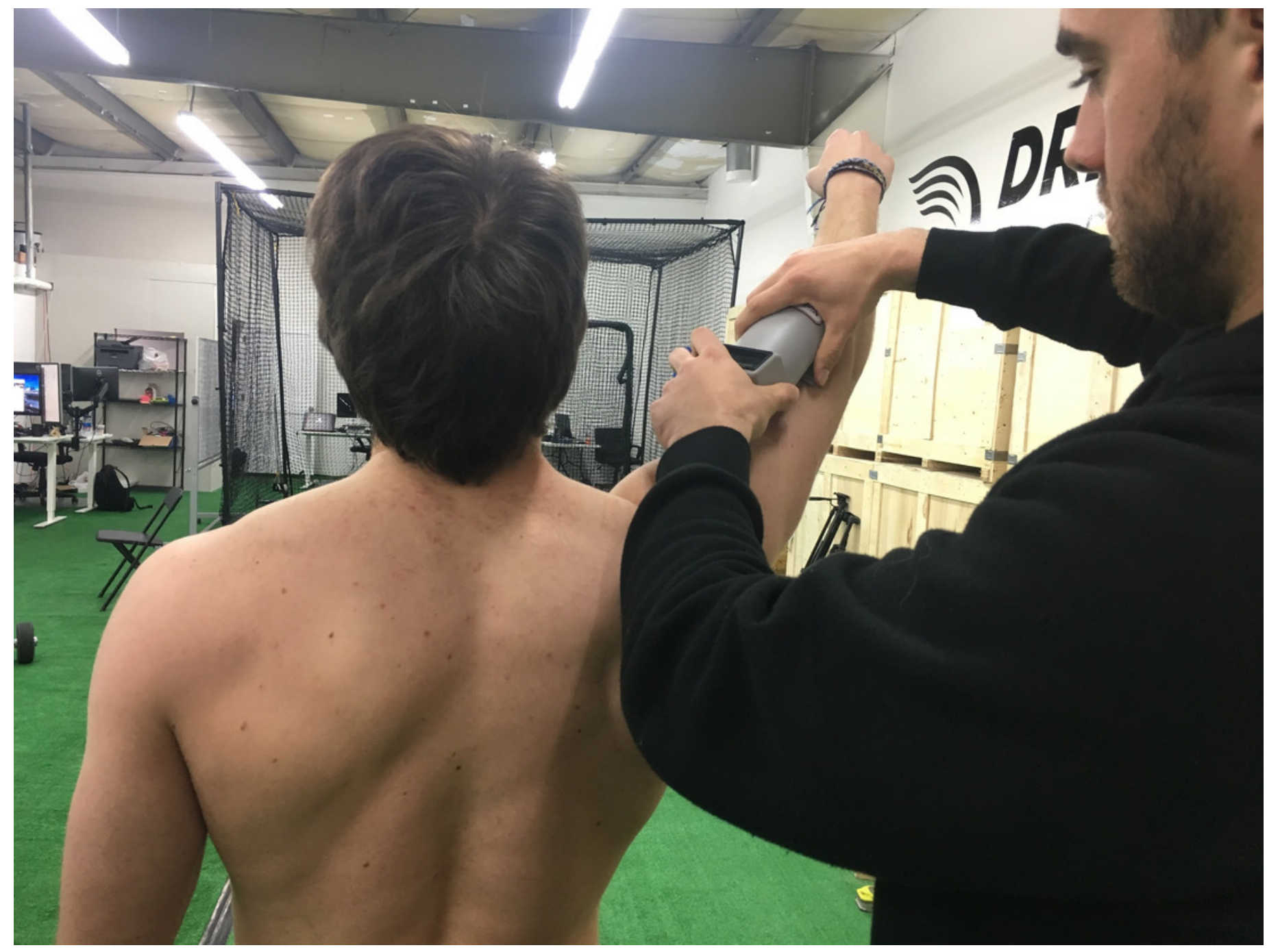


Figure 6

\section{Dynamic Time Warping example}

An example of Dynamic Time Warping as produced by R-Studio.

Photo credit: Alex Caravan

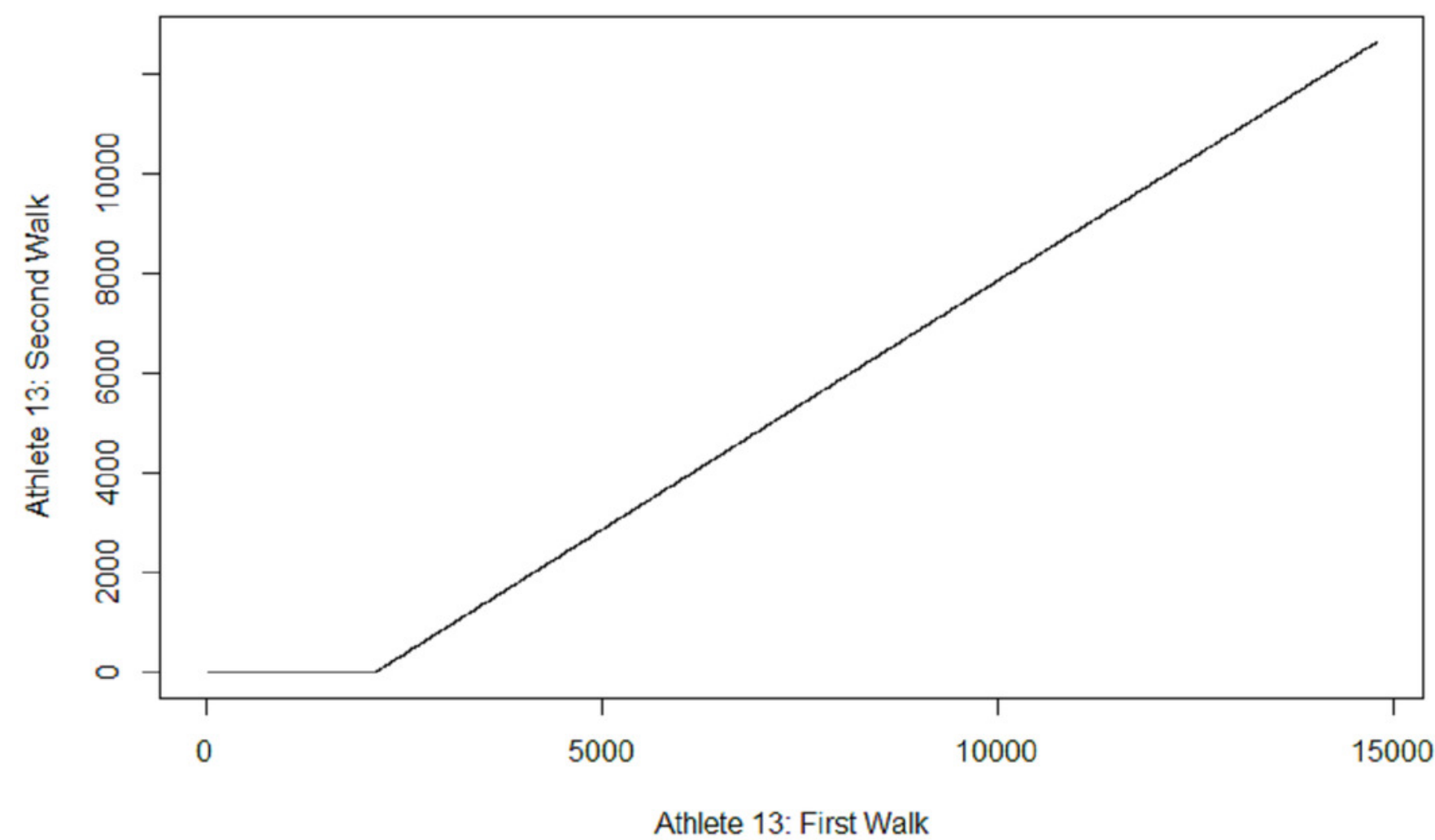




\section{Table 1 (on next page)}

Looking at overall group differences

An analysis of the total group differences in the subject pool.

Photo credit: Alex Caravan 
1

2

3

4

5

645 Degree Mean Frequencies

790 Degree Mean Frequencies

845 Degree Median Frequencies

990 Degree Median Frequencies
Table X: Looking at Overall Group Differences

Mean $(n=33) \quad$ SEM

9.799

0.152

9.354

0.161

0.071

0.003

0.077

0.006

10

11 


\section{Table 2 (on next page)}

\section{Group differences subset by order of walk}

Examining if the order of the posture changes made a significant difference.

Photo credit: Alex Caravan 
1

2

3

4

5

$645\left(^{\circ}\right)$ Walk MNF

9.754

9.202

0.073

0.083

$845\left(^{\circ}\right)$ Walk MDF

$990\left({ }^{\circ}\right)$ Walk MDF

Table Y: Group Differences Subset by Order of Walk

Mean 1st Group (n=17)

SEM

Mean 2nd Group ( $n=16)$

SEM

10 